\title{
Reperfusion strategies in stroke due to isolated cervical internal carotid artery occlusion: systematic review and treatment comparison
}

\author{
Michele Romoli ${ }^{1,2}$ (D) Maria Giulia Mosconi ${ }^{3} \cdot$ Patrizia Pierini $^{3} \cdot$ Andrea Alberti $^{3} \cdot$ Michele Venti $^{3} \cdot$ Valeria Caso $^{4}$. \\ Simone Vidale ${ }^{2}$ - Enrico Maria Lotti ${ }^{2}$ - Marco Longoni ${ }^{4} \cdot$ Paolo Calabresi $^{5,6} \cdot$ Georgios Tsivgoulis $^{7,8}$. \\ Maurizio Paciaroni ${ }^{3}$
}

Received: 1 June 2020 / Accepted: 17 September 2020 / Published online: 10 October 2020

(C) The Author(s) 2020

\begin{abstract}
Introduction Despite intravenous thrombolysis (IVT) and endovascular treatment (EVT) have been demonstrated effective in acute ischemic stroke (AIS) due to large vessel occlusions, there are still no conclusive data to guide treatment in stroke due to cervical internal carotid artery (ICA) occlusion. We systematically reviewed available literature to compare IVT, EVT, and bridging (IVT + EVT) and define optimal treatment.

Methods Systematic review followed predefined protocol (Open-Science-Framework osf.io/bfykj). MEDLINE, EMBASE, and Cochrane CENTRAL were searched. Results were restricted to studies in English, with sample size $\geq 10$ and follow-up $\geq 30$ days. Primary outcomes were favorable outcome $(\mathrm{mRS} \leq 2)$, mortality, and symptomatic intracerebral hemorrhage $(\mathrm{sICH})$, defined according to study original report. Newcastle-Ottawa scale was used for bias assessment.

Results Seven records of 930 screened were included in meta-analysis. Quality of studies was low-to-fair in 5, good in 2. IVT (n $=450)$ did not differ for favorable outcome and mortality compared to EVT $(n=150)$, though having lower rate of sICH $(\mathrm{OR}=$ 0.4, 95\% CI 0.2-0.8). Compared to IVT, bridging (IVT + EVT) was associated with higher rate of favorable outcome $(\mathrm{OR}=2.2$, 95\% CI 1.3-3.7). Compared to EVT, bridging (IVT + EVT) provided higher rate of favorable outcome (OR = 1.9, 95\% CI 1.1$3.4)$, with a marginally increased risk of $\mathrm{SICH}(\mathrm{OR}=2.1,95 \%$ CI $1-4.4)$ but similar mortality rates.

Conclusions Our systematic review highlights that, in acute ischemic stroke associated with isolated cervical ICA occlusion, bridging (IVT + EVT) might lead to higher rate of functional independence at follow-up, without increasing mortality. The low quality of available studies prevents from drawing firm conclusions, and randomized-controlled clinical trials are critically needed to define optimal treatment in this AIS subgroup.
\end{abstract}

Michele Romoli and Maria Giulia Mosconi are First authors

Electronic supplementary material The online version of this article (https://doi.org/10.1007/s10072-020-04735-5) contains supplementary material, which is available to authorized users.

Michele Romoli

michele.romoli@studenti.unipg.it; michele.romoli@auslromagna.it

1 Neurology Clinic, University of Perugia-S. Maria della Misericordia Hospital, Perugia, Italy

2 Neurology Unit, Rimini “Infermi” Hospital-AUSL Romagna, Viale Settembrini 2, 47923 Rimini, Italy

3 Stroke Unit and Division of Cardiovascular Medicine, University of Perugia-S. Maria della Misericordia Hospital, Perugia, Italy

4 Neurology Unit, "M. Bufalini” Hospital-AUSL Romagna, Cesena, Italy
5 Institute of Neurology, Catholic University of Sacred Heart, Roma, Italy

6 Fondazione Policlinico Universitario A. Gemelli IRCCS, Roma, Italy

7 Second Department of Neurology, "Attikon" Hospital School of Medicine, National and Kapodistrian University of Athens, Athens, Greece

8 Department of Neurology, University of Tennessee Health Science Center, Memphis, TN, USA 
Keywords Thrombectomy $\cdot$ Thrombolysis $\cdot$ stroke $\cdot$ Ischemic stroke $\cdot$ Internal carotid artery

\section{Introduction}

Intravenous thrombolysis (IVT) with recombinant tissue plasminogen activator (rtPA) is approved as a first-line treatment for acute ischemic stroke within appropriate time-frame [1]. In recent years, a bulk of literature has accumulated supporting the effectiveness and safety of endovascular treatment (EVT), which has therefore been implemented in current guidelines as bridging (IVT + EVT) or as direct intervention [1].

Previous randomized-controlled clinical trials (RCTs) and observational studies have highlighted differences in the effectiveness of reperfusion strategies depending on occlusion site [2-4]. In particular, strokes due to acute internal carotid artery (ICA) occlusion are associated with poor prognosis [3, 4]. Occlusion of extracranial ICA has very low recanalization rates, so that poor benefit from IVT alone has been postulated $[3,5]$. A previous systematic review highlighted that EVT might provide higher rates of favorable outcome, though associating with significantly higher risk of symptomatic intracerebral hemorrhage (sICH) [4]. However, results were derived from studies with interventions mostly limited to what nowadays is considered a restricted time-window. Moreover, data from studies using IVT before EVT were pooled in EVT group, and ICA site of occlusion varied from cervical to terminal or tandem [4], preventing from drawing meaningful conclusions on the optimal reperfusion strategy.

To date, no completed RCTs exist addressing the specific question of the optimal reperfusion treatment in stroke due to extracranial ICA occlusion. Moreover, despite a vast amount of literature produced on stroke due to large vessel occlusions [6], there are still no conclusive clinical data to guide best treatment approach in extracranial ICA occlusion-related stroke.

Here we provide a systematic analysis of published studies to define clinical outcomes in patients stroke due to occlusion extracranial ICA treated with IVT, EVT, or bridging therapy.

\section{Methods}

\section{Search strategy and selection criteria of the systematic review}

Systematic review followed PRISMA guidelines and protocol registered with Open Science Framework (osf.io/bfykj). MEDLINE, EMBASE, and Cochrane Central Register for Controlled Trials (CENTRAL) were searched up to March 04, 2020 for studies reporting on treatment of acute ischemic stroke due to occlusion of extracranial ICA. Search string included combination of the following terms: (i) "stroke" OR "cerebrovascular diseas*"; (ii) "tissue plasminogen activator" OR "thromboly*" OR "rtPA" OR "alteplase" OR "tenecteplase"; (iii) "endovascular procedure*" OR "endovascular treatment" OR "thrombectomy" OR "aspiration" OR "retrieval"; (iv) "internal carotid artery" OR "proximal carotid" OR "extracranial carotid" OR "cervical carotid" (“*” as catch-term). We restricted results to studies with isolated extracranial ICA occlusion documented by ultrasound (US), computed tomographic angiography (CTA), magnetic resonance angiography (MRA) or digital subtraction angiography (DSA), and with sample size of treated patients $\geq 10$ and follow-up of at least 30 days in order to minimize anecdotal report-related bias [4]. Only studies in English were included. Two researchers independently carried out the search and identified eligible studies. Controversies were resolved by the senior author.

\section{Data extraction, bias assessment, and defined outcomes}

Two authors extracted data from eligible papers, including design, setting, sample size, reperfusion strategy (IVT, EVT, or IVT + EVT). For studies published more than once (i.e., duplicates) and with multiple time-points, we included only the report with the most informative and complete data. Outcomes were (i) favorable outcome, according to study original definition, (ii) mortality, and (iii) sICH. For included studies, successful reperfusion according to in-study definition was extracted as secondary outcome. When specific definition of sICH was not available, we considered as SICH (i) hemorrhages associated with decline in neurological status and (ii) parenchymal hematomas. Studies were excluded from respective analysis if they did not provide data on clinical outcomes predefined. Studies reporting outcomes at $<30$ days were excluded. Three groups of reperfusion strategies were defined (IVT, EVT, or IVT + EVT), with clinical outcomes extracted and attributed according to study reports. Cochrane risk of bias tools and Newcastle-Ottawa Scale (NOS) were used for bias assessment depending on study design [7]. A follow-up of at least 30 days was pre-requisite for study inclusion and therefore defined as a reasonable timing for assessing clinical outcomes in the respective NOS item [7].

\section{Statistical analysis}

Outcome distribution is displayed as binary variable across reperfusion strategies, with count and percentages. Chisquare test was used to compare prevalence of primary and 
secondary outcomes depending on reperfusion strategy. Odds ratio (OR) were used to provide estimate of each treatment effect on predefined outcome vs other treatment paradigms, with logistic function implemented to calculate $95 \%$ confidence intervals and $p$-alue, set as $<0.05$ for significance. Heterogeneity was tested and quantified according to Qstatistics [8]. Outcome rates were meta-analyzed via random-effect modeling due to substantial heterogeneity in study design, treatment, and time-windows. Meta-regression analysis was programmed to evaluate age, NIHSS, onset-toneedle, and onset-to-reperfusion timing. Statistical analysis was performed with R-v3.3.1.

\section{Results}

Overall, 165 records were screened (Fig. 1). After exclusion of reports not providing data on isolated extracranial ICA occlusion or outcome (Supplemental material - Table I), 7 studies were retrieved and included and qualitative and quantitative synthesis (Fig. 1).[2, 3, 9-13]. All studies were observational in nature, 5 of them with retrospective [9-13] and 2 with multicenter prospective design $[2,3]$. None of the studies was randomized or controlled, and treatment-provided followed guidelines available at that moment as well as stroke physician decisions. Bias assessment with NOS highlighted good quality for only 2 studies [2,3], the remaining having low to fair quality (Supplemental material - Table II). Three studies reported on IVT only $[2,11,13]$, while 4 studies reported on multiple reperfusion strategies $[3,9,10,12]$.

Six studies provided data for IVT in acute ischemic stroke due to isolated extracranial ICA occlusion $(n=410)[2,3$, 9-11, 13]. All studies used rtPA for the treatment protocol, except for 1 study which also included patients receiving systemic urokinase [9]. Favorable outcome was defined as modified Rankin Scale (mRS) 0-2 at follow-up (ranging 1 to 3 months), except from one study which used more stringent definition (mRS 0-1) [13]. Pooling data, favorable outcome was reported in $28 \%(n=115)$ after IVT, with sICH happening in $6.1 \%(n=25)$, and mortality rate of $25.1 \%(n=103)$. Similar proportions were confirmed by meta-analysis, with high heterogeneity for mortality $\left(I^{2}=65 \%, p_{\text {heterogeneity }}=\right.$ 0.22 ) and low heterogeneity for functional outcome and
Fig. 1 Study selection flow diagram

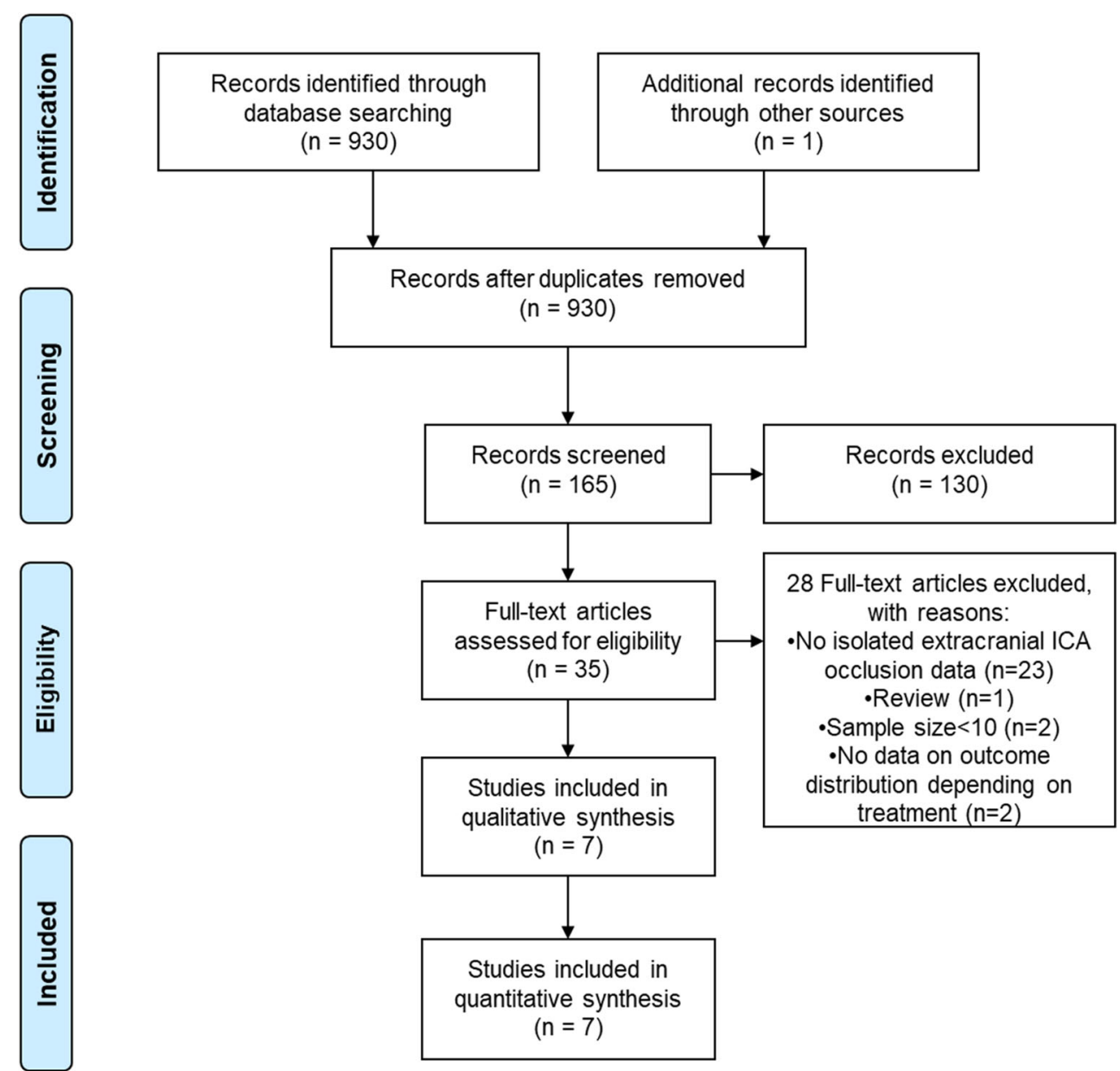




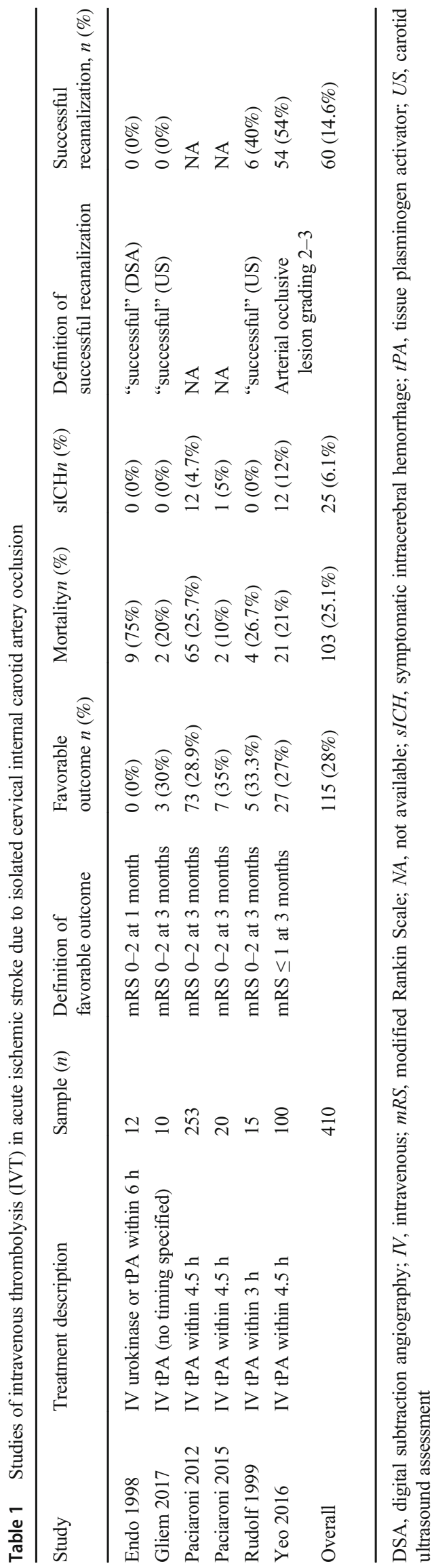

sICH (Supplemental material - Figure I). Secondary outcome had inconsistent definition across studies, and highly variable rates of successful recanalization were reported, with pooled estimate of $14.6 \%$ (Table 1).

Three studies provided data for EVT in acute ischemic stroke due to isolated cervical ICA occlusion $(n=150)$ [3, 9, 12]. EVT highly varied, including IA rtPA, thrombectomy, aspiration, stenting, and angioplasty. Data on clinical outcomes were provided by two studies only $(n=145)$, with favorable outcome (mRS $0-2)$ reported in $31 \%(n=45)$. sICH rate was $13.8 \%(n=20)$, and mortality $23.4 \%(n=$ $34)$. Similar proportions were confirmed by meta-analysis, with high heterogeneity for mortality $\left(I^{2}=86 \%, p_{\text {heterogeneity }}\right.$ $<0.01$ ) and low heterogeneity for functional outcome and sICH (Supplemental material - Figure II). Secondary outcome had inconsistent definition on the two studies reporting recanalization rate, with estimate of $46.2 \%$ (Table 2).

Three studies provided data for bridging treatment (IVT + EVT) $(n=62)[3,10,12]$. Data on clinical outcomes were provided by two studies only $(n=56)[3,10]$, with favorable outcome (mRS 0-2) reported in $46.4 \%(n=26)$, sICH in $25 \%$ $(n=14)$, and mortality in $19.6 \%(n=11)$. Similar proportions were confirmed by meta-analysis, with low heterogeneity (Supplemental material - Figure III). Secondary outcome was inconsistently reported, with estimate of $94.4 \%$ (Table 3).

Comparing pooled estimates of primary and secondary outcomes depending on reperfusion strategy (Table 4), IVT did not differ for favorable outcome, mortality, and successful recanalization compared to EVT, though having lower rate of SICH (OR 0.4, 95\% CI 0.2-0.8). Compared to IVT, bridging (IVT + EVT) was associated with higher rate of favorable outcome (OR 2.2, 95\% CI 1.3-3.7) and SICH (OR 5.1, 95\% CI 2.5-10.5), though not impacting on mortality (OR $0.7,95 \%$ CI $0.4-1.4$ ). Compared to EVT, bridging (IVT + EVT) provided higher rate of favorable outcome (OR 1.9, 95\% CI 1.1-3.4) and successful recanalization (OR 19.8, 95\% CI 7.7-51.4), with a marginally increased risk of sICH (OR 2.1, 95\% CI 1-4.4) but similar mortality rates (Table 4). Compared to IVT alone, any EVT (EVT or IVT + EVT) $(n=201)$ resulted in marginally more frequent favorable outcome (OR 1.4, 95\% CI 1.0-2.0) and higher successful recanalization rates (OR 2.5, 95\% CI 1.4-4.3), though increasing sICH (OR 3.1, 95\% CI 1.8-5.4) (Table 4). Similar results were obtained comparing pooled estimates from random-effect metaanalysis (Supplemental material - Table III), which were partially limited by heterogeneity, sample size, and reporting biases (Supplemental material, Figures I-IV). Number of studies included $(<10)$ precluded meta-regression analysis.

\section{Discussion}

Our analysis suggests that a bridging approach to stroke caused by acute isolated cervical ICA occlusion might confer 


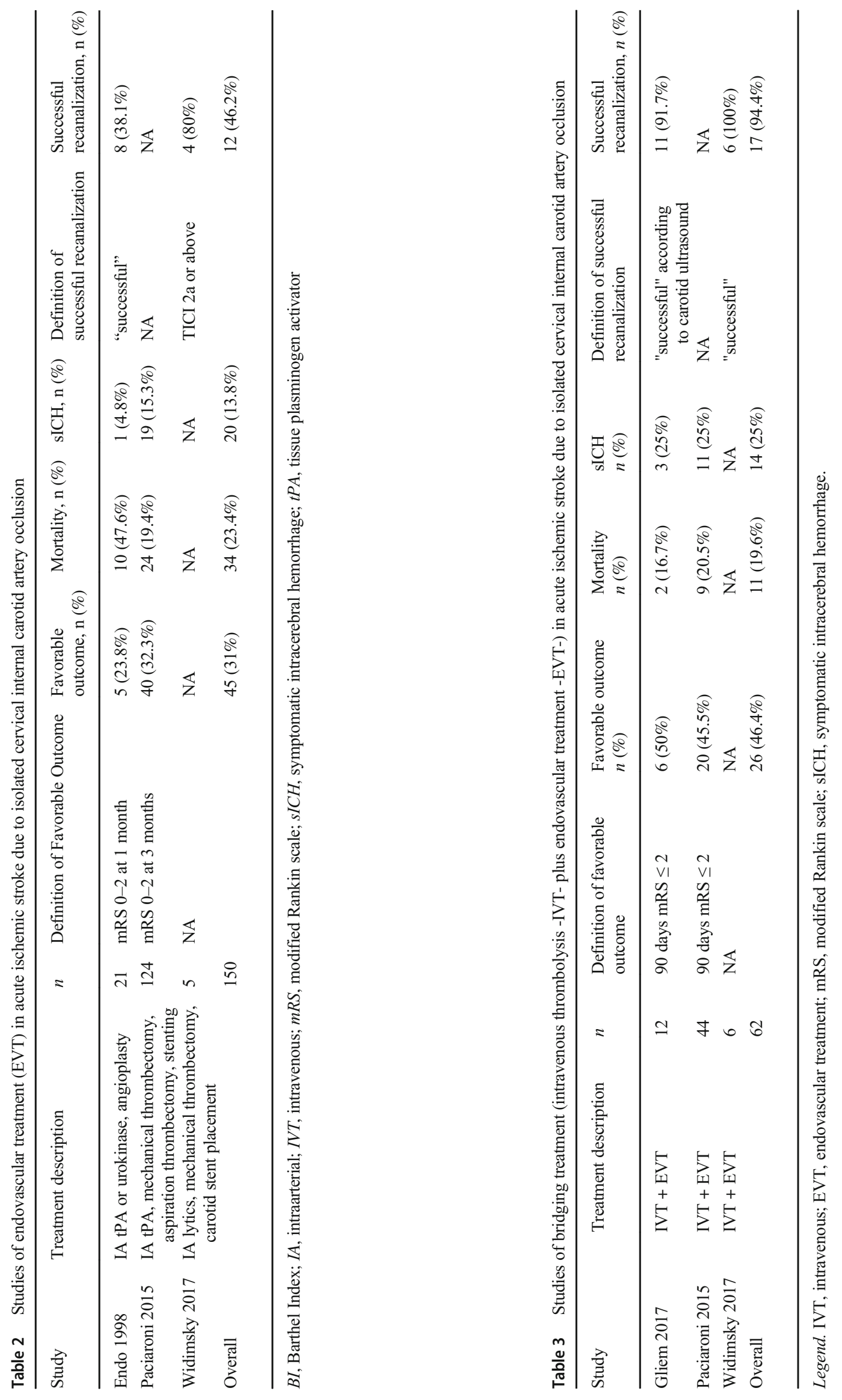


Table 4 Comparison of outcomes depending on reperfusion strategy

\begin{tabular}{|c|c|c|c|c|}
\hline & $\begin{array}{l}\text { EVT } \\
n(\%)\end{array}$ & $\begin{array}{l}\text { IVT } \\
n(\%)\end{array}$ & OR $(95 \% \mathrm{CI})$ & $p$ value \\
\hline Favorable outcome & $45(31 \%)$ & $115(28 \%)$ & $1.2(0.8-1.7)$ & 0.4953 \\
\hline Mortality & $34(23.4 \%)$ & $103(25.1 \%)$ & $0.9(0.6-1.4)$ & 0.6879 \\
\hline sICH & $20(13.8 \%)$ & $25(6.1 \%)$ & $2.5(1.3-4.6)$ & 0.0045 \\
\hline Successful recanalization & $\begin{array}{l}12(46.2 \%) \\
\text { IVT + EVT } \\
n(\%)\end{array}$ & $\begin{array}{l}60(43.8 \%) \\
\text { IVT } \\
n(\%)\end{array}$ & $\begin{array}{l}1.1(0.5-2.3) \\
\text { OR }(95 \% \mathrm{CI})\end{array}$ & $\begin{array}{l}0.8244 \\
p \text { value }\end{array}$ \\
\hline Favorable outcome & $26(46.4 \%)$ & $115(28 \%)$ & $2.2(1.3-3.7)$ & 0.0058 \\
\hline Mortality & $11(19.6 \%)$ & $103(25.1 \%)$ & $0.7(0.4-1.4)$ & 0.3725 \\
\hline sICH & $14(25 \%)$ & $25(6.1 \%)$ & $5.1(2.5-10.5)$ & $<0.0001$ \\
\hline Successful recanalization & $\begin{array}{l}17(94.4 \%) \\
\text { IVT + EVT } \\
n(\%)\end{array}$ & $\begin{array}{l}60(43.8 \%) \\
\mathrm{EVT} \\
n(\%)\end{array}$ & $\begin{array}{l}21.8(10.5-45.2) \\
\text { OR }(95 \% \mathrm{CI})\end{array}$ & $\begin{array}{l}0.0031 \\
p \text { value }\end{array}$ \\
\hline Favorable outcome & $26(46.4 \%)$ & $45(31 \%)$ & $1.9(1.1-3.4)$ & 0.0421 \\
\hline Mortality & $11(19.6 \%)$ & $34(23.4 \%)$ & $0.8(0.4-1.7)$ & 0.5623 \\
\hline sICH & $14(25 \%)$ & $20(13.8 \%)$ & $2.1(1-4.4)$ & 0.0608 \\
\hline Successful recanalization & $\begin{array}{l}17(94.4 \%) \\
\text { All EVT* } \\
n(\%)\end{array}$ & $\begin{array}{l}12(46.2 \%) \\
\text { IVT } \\
n(\%)\end{array}$ & $\begin{array}{l}19.8(7.7-51.4) \\
\text { OR }(95 \% \mathrm{CI})\end{array}$ & $\begin{array}{l}0.0067 \\
p \text { value }\end{array}$ \\
\hline Favorable outcome & $71(35.3 \%)$ & $115(28 \%)$ & $1.4(1-2)$ & 0.067 \\
\hline Mortality & $45(22.4 \%)$ & $103(25.1 \%)$ & $0.9(0.6-1.3)$ & 0.459 \\
\hline sICH & $34(16.9 \%)$ & $25(6.1 \%)$ & $3.1(1.8-5.4)$ & $<0.0001$ \\
\hline Successful recanalization & $29(65.9 \%)$ & $60(43.8 \%)$ & $2.5(1.4-4.3)$ & 0.012 \\
\hline
\end{tabular}

*Includes IVT + EVT and EVT without prior IVT; $C I$, confidence interval; $E V T$, endovascular treatment; IVT, intravenous; $O R$, odds ratio; $s I C H$, symptomatic intracerebral hemorrhage higher rates of favorable outcomes compared to IVT or EVT alone. However, this comes at the cost of a higher risk of sICH, though not impacting on mortality. IVT or EVT yielded similar efficacy outcomes, but EVT was related to higher odds of symptomatic intracranial bleeding.

Our results add to the available literature and refine findings from previous reports on reperfusion strategies in large vessel occlusion. Higher rates of reperfusion and improvement in functional outcome have been reported with bridging treatment compared to IVT alone [6, 14-18]. However, there is still vast debate on the role of IVT before EVT on large vessel occlusion, with a reverberating hypothesis of a potential hazard with IVT as opposed to a $10 \%$ chance of recanalization with IVT only $[6,19]$. Isolated cervical ICA occlusion has been to some extent neglected by RCTs and large-scale studies, with the bulk of the literature addressing, as large vessels, distal ICA, tandem occlusion, and proximal middle cerebral artery segments $[6,19]$. Therefore, the optimal management of acute stroke due to cervical ICA occlusion remains elusive. A previous systematic review on ICA occlusion suggested a possible benefit of EVT over IVT in terms of functional outcome [4]. However, all studies addressing ICA occlusion were pooled together, with consistent variations on occlusion site (cervical, terminus, intracranial) and differences in concomitant intracranial vessel occlusion (tandem), and EVT treatment data were derived merging bridging and EVT alone, therefore limiting the clinical implications of the results [4]. Our results, deriving from a systematic review with predefined protocol and stringent inclusion criteria, highlight that, when facing isolated cervical ICA occlusion, bridging with IVT + EVT might improve functional outcome. Such treatment approach is further supported by the fact that a slight increase in sICH compared to IVT does not lead to higher mortality, possibly suggesting that EVT after IVT adds to the chances of recovery without impacting survival.

However, the results of our systematic review need to be considered in the light of several limitations. First, the quality of studies included is generally low, and prevents from drawing firm conclusions on the optimal reperfusion strategy in patients with stroke due to isolated cervical ICA occlusion. Given current guidelines, supporting the use of bridging in large vessel occlusion within appropriate timing, it would be reasonable to explore if differences in functional outcome can be confirmed comparing direct EVT vs bridging treatment in an ad-hoc designed trial. Unfortunately, even latest trials (e.g., DIRECT-MT [20]) did not include patients with isolated cervical occlusion. Given an absolute difference in good functional outcome of $15.4 \%$, we anticipate that a total sample size 
of 312 patients with acute ischemic stroke due to isolated cervical ICA occlusion allocated 1:1 in equal groups would yield a power of $80 \%$ to detect a significant $(p<0.05) \mathrm{im}$ provement in favorable outcome, defined as mRS $0-2$ at 3 months. As a second limitation of the study, several factors might have influenced the benefit of each treatment approach. Indeed, studies slightly differed in terms of age, NIHSS score at admission, and treatment timing. In this study, we were unable to adjust for such potential bias with meta-regression due to the small number of available papers. However, it is reasonable to hypothesize that the earlier the treatment the higher the benefit, as cervical ICA occlusion might associate with broad hemispheric penumbra. To this extent, the higher rates of functional outcomes in studies with 3-4.5 h treatment time window vs those with longer timing [9] seems to corroborate the hypothesis, although the need for RCTs on this population remains crystal-clear. Third, we did not include surgical approach, such as embolectomy or endarterectomy approach, in the hyperacute setting of isolated cervical carotid artery occlusion. However, reports on such approach are rather isolated, and need refinement through observational and randomized studies. As a fourth limitation, EVT technique highly varied across studies, and has definitely expanded in the last decade. Therefore, we might suppose that sICH could have been overestimated, given the use of IA thrombolytics, and that, with development of more effective devices, direct EVT could provide even higher rates of recanalization and lower risk of bleeding within narrow onset-tointervention windows. Fifth, only few studies provided data on isolated cervical ICA occlusion treatment, no mRS shift analysis was available to interpolate data from different studies, and overall sample size was too restricted to allow firm generalizability of results. To this extent, it seems mandatory, in the near future as well as in the long-term, to promote full data sharing, even in the form of global repositories or supplementary material, to help limiting reporting bias. Finally, we only report unadjusted associations of EVT, IVT and bridging therapy with safety and efficacy outcomes due to lack of detailed data.

\section{Conclusion}

Overall, our results highlight that, in acute ischemic stroke associated with isolated cervical ICA occlusion, compared to IVT or EVT alone, bridging (IVT + EVT) might lead to higher rate of functional independence at follow-up, without increasing mortality. The analysis is limited by quality and numerosity of studies, which prevented meta-regression analysis. Larger trials are critically needed to define optimal treatment for patients with stroke due to isolated cervical ICA occlusion.
Acknowledgements Open access funding provided by Università degli Studi di Perugia within the CRUI-CARE Agreement.

\section{Compliance with ethical standards}

Conflict of interest The authors declare that they have no conflict of interest.

Ethical approval Not needed

Consent to participate/publication Not needed

Data sharing and data accessibility Dataset used for analysis can be shared upon reasonable request to the corresponding author.

Open Access This article is licensed under a Creative Commons Attribution 4.0 International License, which permits use, sharing, adaptation, distribution and reproduction in any medium or format, as long as you give appropriate credit to the original author(s) and the source, provide a link to the Creative Commons licence, and indicate if changes were made. The images or other third party material in this article are included in the article's Creative Commons licence, unless indicated otherwise in a credit line to the material. If material is not included in the article's Creative Commons licence and your intended use is not permitted by statutory regulation or exceeds the permitted use, you will need to obtain permission directly from the copyright holder. To view a copy of this licence, visit http://creativecommons.org/licenses/by/4.0/.

\section{References}

1. Powers WJ, Rabinstein AA, Ackerson T, Adeoye OM, Bambakidis NC, Becker K, Biller J, Brown M, Demaerschalk BM, Hoh B, Jauch EC, Kidwell CS, Leslie-Mazwi TM, Ovbiagele B, Scott PA, Sheth KN, Southerland AM, Summers DV, Tirschwell DL (2018) Guidelines for the early management of patients with acute ischemic stroke: a guideline for healthcare professionals from the American Heart Association/American Stroke Association. Stroke 49:e46-e110. https://doi.org/10.1161/STR.0000000000000158

2. Paciaroni M, Balucani C, Agnelli G, Caso V, Silvestrelli G, Grotta JC, Demchuk AM, Sohn SI, Orlandi G, Leys D, Pezzini A, Alexandrov AV, Silvestrini M, Fofi L, Barlinn K, Inzitari D, Ferrarese C, Tassi R, Tsivgoulis G, Consoli D, Baldi A, Bovi P, Luda E, Galletti G, Invernizzi P, DeLodovici ML, Corea F, del Sette M, Monaco S, Marcheselli S, Alberti A, Venti M, Acciarresi M, D'Amore C, Macellari F, Lanari A, Previdi P, Gonzales NR, Pandurengan RK, Vahidy FS, Sline M, Bal SS, Chiti A, Gialdini G, Dumont F, Cordonnier C, Debette S, Padovani A, Cerqua R, Bodechtel U, Kepplinger J, Nesi M, Nencini P, Beretta S, Trentini C, Martini G, Piperidou C, Heliopoulos I, D'Anna S, Cappellari M, Donati E, Bono G, Traverso E, Toni D (2012) Systemic thrombolysis in patients with acute ischemic stroke and internal carotid artery occlusion: the ICARO study. Stroke 43:125130. https://doi.org/10.1161/STROKEAHA.111.630624

3. Paciaroni M, Inzitari D, Agnelli G, Caso V, Balucani C, Grotta JC, Sarraj A, Sung-Il S, Chamorro A, Urra X, Leys D, Henon H, Cordonnier C, Dequatre N, Aguettaz P, Alberti A, Venti M, Acciarresi M, D'Amore C, Zini A, Vallone S, Dell'Acqua ML, Menetti F, Nencini P, Mangiafico S, Barlinn K, Kepplinger J, Bodechtel U, Gerber J, Bovi P, Cappellari M, Linfante I, Dabus G, Marcheselli S, Pezzini A, Padovani A, Alexandrov AV, Shahripour RB, Sessa M, Giacalone G, Silvestrelli G, Lanari A, 
Ciccone A, de Vito A, Azzini C, Saletti A, Fainardi E, Orlandi G, Chiti A, Gialdini G, Silvestrini M, Ferrarese C, Beretta S, Tassi R, Martini G, Tsivgoulis G, Vasdekis SN, Consoli D, Baldi A, D’Anna S, Luda E, Varbella F, Galletti G, Invernizzi P, Donati E, de Lodovici ML, Bono G, Corea F, Sette MD, Monaco S, Riva M, Tassinari T, Scoditti U, Toni D (2015) Intravenous thrombolysis or endovascular therapy for acute ischemic stroke associated with cervical internal carotid artery occlusion: the ICARO-3 study. J Neurol 262:459-468. https://doi.org/10.1007/s00415-014-7550-1

4. Mokin M, Kass-Hout T, Kass-Hout O, Dumont TM, Kan P, Snyder KV, Hopkins LN, Siddiqui AH, Levy EI (2012) Intravenous thrombolysis and endovascular therapy for acute ischemic stroke with internal carotid artery occlusion: A systematic review of clinical outcomes. Stroke 43:2362-2368. https://doi.org/10.1161/ STROKEAHA.112.655621

5. Linfante I, Llinas RH, Selim M, Chaves C, Kumar S, Parker RA, Caplan LR, Schlaug G (2002) Clinical and vascular outcome in internal carotid artery versus middle cerebral artery occlusions after intravenous tissue plasminogen activator. Stroke 33:2066-2071. https://doi.org/10.1161/01.STR.0000021001.18101.A5

6. Tsivgoulis G, Katsanos AH, Schellinger PD, Köhrmann M, Varelas P, Magoufis G, Paciaroni M, Caso V, Alexandrov AW, Gurol E, Alexandrov AV (2018) Successful reperfusion with intravenous thrombolysis preceding mechanical thrombectomy in large-vessel occlusions. Stroke 49:232-235. https://doi.org/10.1161/ STROKEAHA.117.019261

7. Luchini C, Stubbs B, Solmi M et al (2017) Assessing the quality of studies in meta-analyses: advantages and limitations of the Newcastle Ottawa Scale. World J Metaanal 5:80. https://oi.org/ 10.13105/wjma.v5.i4.80

8. Higgins JPT, Thompson SG (2002) Quantifying heterogeneity in a meta-analysis. Stat Med 21:1539-1558. https://doi.org/10.1002/ $\operatorname{sim} .1186$

9. Endo S, Kuwayama N, Hirashima Y, Akai T, Nishijima M, Takaku A (1998) Results of urgent thrombolysis in patients with major stroke and atherothrombotic occlusion of the cervical internal carotid artery. Am J Neuroradiol 19:1169-1175

10. Gliem M, Lee JI, Barckhan A, Turowski B, Hartung HP, Jander S (2017) Outcome and treatment effects in stroke associated with acute cervical ICA occlusion. PLoS One 12:1-8. https://doi.org/ 10.1371/journal.pone. 0170247

11. Rudolf J, Neveling M, Grond M, Schmulling S, Stenzel C, Heiss WD (1999) Stroke following internal carotid artery occlusion - A contra-indication for intravenous thrombolysis? Eur J Neurol 6:5155. https://doi.org/10.1046/j.1468-1331.1999.610051.x

12. Widimsky P, Koznar B, Peisker T, Vasko P, Rohac F, Vavrova J, Kroupa J, Stetkarova I (2017) Feasibility and safety of direct catheter-based thrombectomy in the treatment of acute ischaemic stroke. Cooperation among cardiologists, neurologists and radiologists. Prospective registry Prague-16. EuroIntervention 13:131136. https://doi.org/10.4244/EIJ-D-16-00979

13. Yeo LLL, Kong WY, Paliwal P, Teoh HL, Seet RC, Soon D, Rathakrishnan R, Ong V, Lee TH, Wong HF, Chan BPL, Leow WK, Yuan C, Ting E, Gopinathan A, Tan BYQ, Sharma VK (2016) Intravenous thrombolysis for acute ischemic stroke due to cervical internal carotid artery occlusion. J Stroke Cerebrovasc Dis
25:2423-2429. https://doi.org/10.1016/j.jstrokecerebrovasdis. 2016.06.014

14. Katsanos AH, Malhotra K, Goyal N, Arthur A, Schellinger PD, Köhrmann M, Krogias C, Turc G, Magoufis G, Leys D, Ahmed N, Khatri P, Goyal M, Alexandrov AV, Tsivgoulis G (2019) Intravenous thrombolysis prior to mechanical thrombectomy in large vessel occlusions. Ann Neurol 86:395-406. https://doi.org/ 10.1002/ana.25544

15. Katsanos AH, Tsivgoulis G (2019) Is intravenous thrombolysis still necessary in patients who undergo mechanical thrombectomy? Curr Opin Neurol 32:3-12. https://doi.org/10.1097/WCO. 0000000000000633

16. Goyal N, Tsivgoulis G, Frei D, Turk A, Baxter B, Froehler MT, Mocco J, Pandhi A, Zand R, Malhotra K, Hoit D, Elijovich L, Loy D, Turner RD, Mascitelli J, Espaillat K, Katsanos AH, Alexandrov AW, Alexandrov AV, Arthur AS (2018) Comparative safety and efficacy of combined ivt and $\mathrm{mt}$ with direct $\mathrm{mt}$ in large vessel occlusion. Neurology 90:e1274-e1282. https://doi.org/10.1212/ WNL.0000000000005299

17. Wollenweber FA, Tiedt S, Alegiani A, Alber B, Bangard C, Berrouschot J, Bode FJ, Boeckh-Behrens T, Bohner G, Bormann A, Braun M, Dorn F, Eckert B, Flottmann F, Hamann GF, Henn KH, Herzberg M, Kastrup A, Kellert L, Kraemer C, Krause L, Lehm M, Liman J, Lowens S, Mpotsaris A, Papanagiotou P, Petersen M, Petzold GC, Pfeilschifter W, Psychogios MN, Reich A, von Rennenberg R, Röther J, Schäfer JH, Siebert E, Siedow A, Solymosi L, Thonke S, Wagner M, Wunderlich S, Zweynert S, Nolte CH, Gerloff C, Thomalla G, Dichgans M, Fiehler J (2019) Functional outcome following stroke thrombectomy in clinical practice. Stroke 50:2500-2506. https://doi.org/10.1161/ STROKEAHA.119.026005

18. Chalos V, LeCouffe NE, Uyttenboogaart M et al (2019) Endovascular treatment with or without prior intravenous alteplase for acute ischemic stroke. J Am Heart Assoc 8:1-15. https://doi. org/10.1161/JAHA.118.011592

19. Vidale S, Romoli M, Consoli D, Agostoni EC (2020) Bridging versus Direct Mechanical Thrombectomy in Acute Ischemic Stroke: A Subgroup Pooled Meta-Analysis for Time of Intervention, Eligibility, and Study Design. Cerebrovasc Dis 24: $1-10$

20. Yang P, Zhang Y, Zhang L, Zhang Y, Treurniet KM, Chen W, Peng Y, Han H, Wang J, Wang S, Yin C, Liu S, Wang P, Fang Q, Shi H, Yang J, Wen C, Li C, Jiang C, Sun J, Yue X, Lou M, Zhang M, Shu H, Sun D, Liang H, Li T, Guo F, Ke K, Yuan H, Wang G, Yang W, Shi H, Li T, Li Z, Xing P, Zhang P, Zhou Y, Wang H, Xu Y, Huang Q, Wu T, Zhao R, Li Q, Fang Y, Wang L, Lu J, Li Y, Fu J, Zhong X, Wang Y, Wang L, Goyal M, Dippel DWJ, Hong B, Deng B, Roos YBWEM, Majoie CBLM, Liu J (2020) Endovascular thrombectomy with or without intravenous alteplase in acute stroke. N Engl J Med 382:1981-1993. https:// doi.org/10.1056/NEJMoa2001123

Publisher's note Springer Nature remains neutral with regard to jurisdictional claims in published maps and institutional affiliations. 